\title{
A Rare and Interesting Case of Rhinofacial Zygomycosis Due to Conidiobolus Coronatus
}

Jayasena K. D. N. U. D., Dayarathne S. M.

\begin{abstract}
We report a 53-year-old farmer, with right sided epiphorea, headache and nasal obstruction for 2 months. FONL revealed a growth beneath right inferior turbinate. He was diagnosed to have Rhinofacial Zygomycosis due to Conidiobolus coronata and treated with oral Itraconazole. He improved and is still on follow up.
\end{abstract}

\section{Introduction}

Zygomycosis due to Conidiobolus coronata is a rare disease which mimic signs and symptoms of a tumour of the region. It is a sporadic infection of subcutaneous tissue seen in tropical areas off south east Asia, Africa and central America. We report one such rare case with a different presentation.

\section{Case report}

We present a 58 years old male farmer who cultivates both paddy and chena from Embilipitiya Sri Lanka. He has presented to our unit with increased tearing (Epiphorea) from the right eye, right sided mild throbbing type headache and right sided nasal congestion.

He had similar symptoms 2 months ago and was treated by a general practitioner with nasal steroid drops and a course of antibiotics. Symptoms got worse 1 week after.

He underwent Fiber optic nasolaryngoscopy (FONL) and was found to have a growth below right inferior turbinate. A biopsy was taken from the growth and it revealed an inflammatory lesion but, fungal infection could not be ruled out. Computer Tomography (CT) scan was done and it showed a right side inferior turbinate mass.

Surgery was planned. Informed written consent was taken explaining risks. A Sub labial incision was made and a degloving approach was opted. A subcutaneous growth was noticed over medial aspect of the right maxillary sinus. Biopsy was taken and sent for fungal studies.

Direct smears suggested a strong possibility of Conidiobolus coronata and it was cultured. Culture reports confirmed the diagnosis. Other basic investigations such as full blood count, ESR and CRP were normal.

Itraconazole 200mg bd doze was started and within 1 week, the patient improved clinically. He is still on follow-up.

\section{Discussion.}

Zygomycosis is a fungal infection due to zygomycetes group of fungi, in the facial region. It has two orders, Mucorales and Entomophthorales. Mucorales causes a more severe invasive form of fungal infection characterized by vascular thrombi and necrotic lesions mainly in immunocompromised patients. Entomophthorales has two types, one caused by Basldlobolus ranarum and the other by Conidiobolus coronata ${ }^{1}$. Conidiobolus produces swelling of the nose, cheeks and lips. The nasofacial deformity that results have led to the term rhinofacial mycosis.

Human infection with Conidiobolus species most commonly occurs as a chronic rhinofacial silicosis in otherwise healthy hosts ${ }^{2}$.The infection can be transmitted by insect bites or trans epidermal inoculation with contaminated vegetable matter. Traumatic implantation may also play a role ${ }^{3}$.

The first human infection was reported by Lie Klan Joe in Indonesia in $1960^{4}$. The infection usually affects the age group, 20-50 years. They are ten times more common in males and predominantly affect agricultural workers. The fungus can survive in soils and dried vegetables 
for a long period of time.

The usual presentation of conidiobolomycosis in humans is swelling over the nose. The lesions are smooth, rounded and firmly attached to the underlying tissue, but do not involve the bone. The overlying skin remains intact ${ }^{5}$. The disease starts as a submucosal granuloma in the inferior turbinate, spreads to the nasal sinuses, upper lip, forehead and cheek and may produce extensive facial deformity 6 .

The swelling may be severe enough to obstruct vision, cause Dysphagia and stridor. Walker et al reported a disseminated case with blood vessel invasion in a renal transplant patient ${ }^{7}$.

It is characterized histopathologically by the presence of hyphae surrounded by an eosinophilic halo, the so-called SplendoreHoeppli phenomenon ${ }^{8}$. It is seen in a field of chronic inflammation and found in all cases of Entomophthorales but not in Mucorales. Vascular thrombi or extensive necrosis cannot be seen?

Multiple treatment options are available. The drug of choice for this condition is amphotencin $\mathrm{B}^{10}$. Other options available are ketokonazole and fluconazole. Pottassium iodide is being used by some authors but the exact mechanism of action is unknown. Some authors have found a combination of trimethoprim and sulphamethoxazole, useful. Treatment should be continued for at least one month after the lesions have cleared. Medical treatment may have to be combined with surgical correction of the sequelae of the disease which can affect facial appearance.

To conclude, even though this rare disease that usually presents with facial swelling, in early stages it can present with epiphorea with nasal congestion. It is important to consider the occupational history in such a presentation. Diagnosis takes some time to confirm, but on the experience of the microbiologist, early suspicious is possible and treatment can be started. Ketoconazole is good to start as a first line treatment till definite diagnosis is available as it is a relatively safer drug. Early diagnosis and early treatment will minimize unnecessary surgical intervention and disfigurement due to advanced disease.

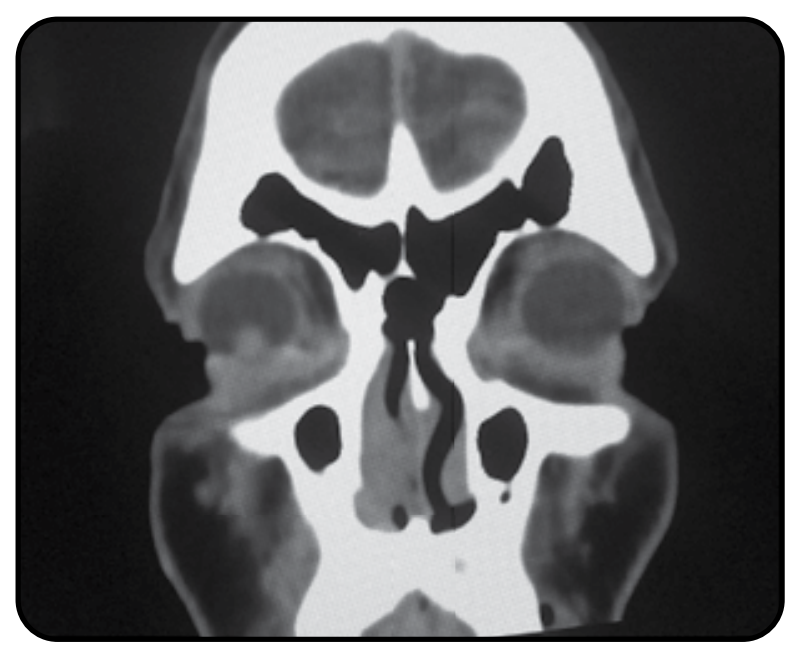

Fig 1- CT Coronal section

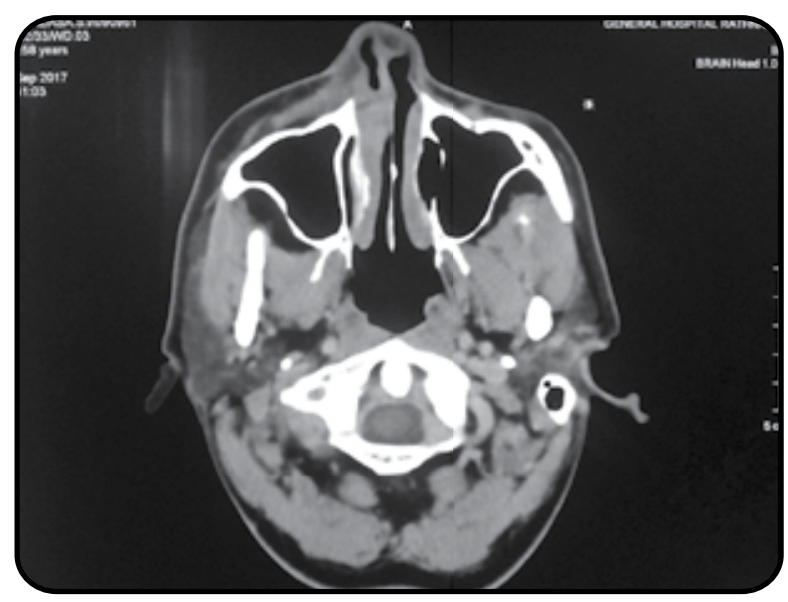

Fig 2- CT Axial section.jpg 


\section{References}

1. BlltzerA, LawsonW.Fungal infections of the headand neck In Otolarvng Clinics of NorthAmenca, Vol 26, WB Sanders Company, pp $1062-1063 ;(1993)$

2. Kwon-Chung KJ, Bennett JE. Medical Mycology. 1992:447-63.

3. zgastrointestinal zygomycosis. Journal of Clinical Microbiology 2001,39(6):2360-2363

4. Nathan,MD, Keller,APJr, Lerner,C J, Jefferson. C D. (1982) Entomophthoralesmlecnon of the maxfllofaclal region Laryngoscope 92 767-769

5. Mathew M. Thomas, Sumangala M. Bal, ChitraJayaprakash, P. Jose, RejeeEbenezer. Julie A. Ribes, Carolyn L.Vanover-Sams and Doris J. Baker. Zygomycetes in HumanDisease. Clin. Microbiol. Rev.2000, 13(2):236,290\} Rhinoentomophthoromycosis. Indian J DermatolVenereolLeprol July - Aug 2006; 72 (4):296 - 299]

6. DawdsonT, Sanders R, (1985) The surgical correcnon of residual facial disfigurement produced by RhlnophycomycosesEntomophthorae A case report, BrmshlournalotPlasnc Surgery 3897 100
7. Isa-Isa $\mathrm{R}$, Arenas $\mathrm{R}$, Fernández RF, Isa MRhinofacialconidiobolomycosis (entomophthoramycosis).Clin Dermatol. 2012 Jul-Aug;30(4):409-12. doi: 10.1016/j. clindermatol.2011.09.012.

8. Wllhams,A ,O (1969) Pathology olPhycomycoses due to Entomophthora and Basldlobolus species Archives of Pathology 86 113-120

9. Shetty D, Rai R, Shetty S, JagdishChandra $\mathrm{K}$, HemaKiniH,RhinofacialZygomycosis - A Rare and Interesting Case Volume 4 Issue 4 2014 ,

10. TaylorGD,Sekhon A S, ]yrrel D L J,Golsand G,(1987)" Rhinofacialzygomycosls caused by Conadloboluscoronatus A case report including invitro sensitivity to antimycotic agents American Journal of tropical Medicine and Hygiene $\sim 6$ 398- 401 